PROCEEDINGS OF THE

AMERICAN MATHEMATICAL SOCIETY

Volume 134, Number 10, October 2006, Pages 2817-2822

S 0002-9939(06)08336-5

Article electronically published on April 11, 2006

\title{
TWO CHARACTERIZATIONS OF PURE INJECTIVE MODULES
}

\author{
KAMRAN DIVAANI-AAZAR, MOHAMMAD ALI ESMKHANI, AND MASSOUD TOUSI
}

(Communicated by Bernd Ulrich)

\begin{abstract}
Let $R$ be a commutative ring with identity and $D$ an $R$-module. It is shown that if $D$ is pure injective, then $D$ is isomorphic to a direct summand of the direct product of a family of finitely embedded modules. As a result, it follows that if $R$ is Noetherian, then $D$ is pure injective if and only if $D$ is isomorphic to a direct summand of the direct product of a family of Artinian modules. Moreover, it is proved that $D$ is pure injective if and only if there is a family $\left\{T_{\lambda}\right\}_{\lambda \in \Lambda}$ of $R$-algebras which are finitely presented as $R$-modules, such that $D$ is isomorphic to a direct summand of a module of the form $\prod_{\lambda \in \Lambda} E_{\lambda}$, where for each $\lambda \in \Lambda, E_{\lambda}$ is an injective $T_{\lambda}$-module.
\end{abstract}

\section{INTRODUCTION}

Throughout this paper, let $R$ denote a commutative ring with identity, and all modules are assumed to be unitary. The notion of injective modules has a substantial role in algebra. There are several generalizations of this notion. One of them is the notion of pure injective modules. An $R$-monomorphism $f: M \longrightarrow N$ is said to be pure if for any $R$-module $L$, the map $f \otimes i d_{L}: M \otimes_{R} L \longrightarrow N \otimes_{R} L$ is injective. An $R$-module $D$ is said to be pure injective if for any pure homomorphism $f: M \longrightarrow N$, the induced homomorphism $\operatorname{Hom}_{R}(N, D) \longrightarrow \operatorname{Hom}_{R}(M, D)$ is surjective. In model theory the notion of pure injective modules is more useful than that of injective modules. Also, there are some excellent applications of this notion in the theory of flat covers. Thus, this notion has attained more notice in recent years. For a survey on pure injective modules, we refer the reader to 8,3 and 9 .

Our aim in this paper is to present two characterizations of pure injective modules. First, we show that a pure injective $R$-module $D$ is isomorphic to a direct summand of the direct product of a family of finitely embedded $R$-modules. Then we deduce our first characterization that says that over a Noetherian ring $R$ an $R$-module $D$ is pure injective if and only if $D$ is isomorphic to a direct summand of the direct product of a family of Artinian modules.

Let $E$ denote an injective cogenerator of the ring $R$. Let $D$ be an $R$-module. We show that $D$ is pure injective if and only if there is a family $\left\{T_{\lambda}\right\}_{\lambda \in \Lambda}$ of $R$ algebras which are finitely presented as $R$-modules, such that $D$ is isomorphic to a

Received by the editors December 16, 2004 and, in revised form, April 21, 2005.

2000 Mathematics Subject Classification. Primary 13E10, 13C05.

Key words and phrases. Pure injective modules, injective cogenerators, finitely embedded modules, finitely presented modules.

This research was supported in part by a grant from IPM (No. 83130115).

(C)2006 American Mathematical Society 
direct summand of $\prod_{\lambda \in \Lambda} \operatorname{Hom}_{R}\left(T_{\lambda}, E\right)$. As an immediate consequence, it follows that $D$ is pure injective if and only if there are an injective $R$-module $E^{\prime}$ and an $R$-module $L$, which is the direct sum of a family of finitely presented $R$-modules such that $D$ is isomorphic to a direct summand of $\operatorname{Hom}_{R}\left(L, E^{\prime}\right)$. Finally, we will deduce our second characterization of pure injective modules which asserts that $D$ is pure injective if and only if there is a family $\left\{T_{\lambda}\right\}_{\lambda \in \Lambda}$ of $R$-algebras which are finitely presented as $R$-modules, such that $D$ is isomorphic to a direct summand of a module of the form $\prod_{\lambda \in \Lambda} E_{\lambda}$, where for each $\lambda \in \Lambda, E_{\lambda}$ is an injective $T_{\lambda}$-module.

\section{The Results}

First, we need to recall a definition and bring some lemmas.

Definition 2.1. An $R$-module $M$ is called cocyclic if $M$ is isomorphic to a submodule of the injective envelope of a simple module (see [5, page 4]). We say that an $R$-module $M$ is finitely embedded if $M$ is isomorphic to a submodule of the injective envelope of the direct sum of finitely many simple modules.

The definition of the dual notion of "finitely generated" is also given separately in 7 and 1]. In the following lemma, we show that those definitions are equivalent to the above definition. In the sequel, for an $R$-module $M$, let $\mathrm{E}(M)$ denote its injective envelope.

Lemma 2.2. Let $M$ be a nonzero R-module. Then the following are equivalent:

i) $M$ is finitely embedded.

ii) There are simple $R$-modules $S_{1}, S_{2}, \ldots, S_{t}$ such that $\mathrm{E}(M)=\mathrm{E}\left(S_{1}\right) \oplus \cdots \oplus$ $\mathrm{E}\left(S_{t}\right)$.

iii) The socle of $M$ is finitely generated, and $M$ is an essential extension of its socle.

iv) For any family $\left\{M_{i}\right\}_{i \in I}$ of submodules of $M$, the intersection $\bigcap_{i \in I} M_{i}$ is nonzero, whenever the intersection of any finite number of $M_{i}$ 's is nonzero.

Proof. i) $\Leftrightarrow$ ii) and ii) $\Leftrightarrow$ iii) follows by [7, Proposition 3.20] and 7, Proposition 3.18 ], respectively. The equivalence iii) $\Leftrightarrow$ iv) follows by [1, Proposition 10.7].

Lemma 2.3. Let $M$ be a nonzero $R$-module. Then $M$ is cocyclic if and only if the intersection of all nonzero submodules of $M$ is nonzero.

Proof. First, assume that $M$ is cocyclic. Then, there is a simple $R$-module $S$ such that $M$ is a submodule of $\mathrm{E}(S)$. Since $\mathrm{E}(S)$ is an essential extension of $S$, it follows that every nonzero submodule of $M$ contains $S$.

Conversely, assume that there is a nonzero element $c \in M$ such that $c$ belongs to all nonzero submodules of $M$. Then, it is routine to check that $R c$ is simple and that $M$ is an essential extension of $R c$. Hence $M$ can be naturally embedded in $\mathrm{E}(R c)$.

Lemma 2.4. Let $M$ be an $R$-module. For any finitely generated $R$-module $N$ and any $0 \neq \alpha \in M \otimes_{R} N$, there exists a finitely embedded quotient module $L$ of $M$ such that the image of $\alpha$ under the natural homomorphism $M \otimes_{R} N \longrightarrow L \otimes_{R} N$ is nonzero.

Proof. Assume that $N$ is generated by $\left\{n_{1}, n_{2}, \ldots, n_{s}\right\}$. Let $F$ be a free $R$-module of rank $s$. Suppose that $\left\{x_{1}, x_{2}, \ldots, x_{s}\right\}$ is a basis for $F$ and that $\rho: F \longrightarrow N$ is the 
natural epimorphism defined by $\sum_{i=1}^{s} r_{i} x_{i} \mapsto \sum_{i=1}^{s} r_{i} n_{i}$. Let $K$ denote the kernel of $\rho$ and $\mu: K \longrightarrow F$ denote the inclusion map. Assume $\beta=\sum_{i=1}^{s}\left(m_{i} \otimes x_{i}\right) \in M \otimes_{R} F$ is such that $\left(i d_{M} \otimes \rho\right)(\beta)=\alpha$. Let $\Sigma$ denote the set of all submodules $P$ of $M \otimes_{R} F$ such that $i m\left(i d_{M} \otimes \mu\right) \subseteq P$ and $\beta \notin P$. Since $\beta \notin i m\left(i d_{M} \otimes \mu\right)$, it follows that $\Sigma$ is not empty. Clearly, $(\Sigma, \subseteq)$ satisfies the assumptions of Zorn's Lemma, and so $\Sigma$ possesses a maximal element, say $Q$. Lemma 2.3 yields that $\left(M \otimes_{R} F\right) / Q$ is cocyclic. Fix $1 \leq i \leq s$, and let $f_{i}: M \longrightarrow M \otimes_{R} F$ be the homomorphism defined by $m \mapsto m \otimes x_{i}$. Let $f_{i}^{*}: M / f_{i}^{-1}(Q) \longrightarrow\left(M \otimes_{R} F\right) / Q$ denote the induced monomorphism. It follows that $M / f_{i}^{-1}(Q)$ is cocyclic. Let $L=M / \bigcap_{i=1}^{s} f_{i}^{-1}(Q)$. Since $\bigoplus_{i=1}^{s} M / f_{i}^{-1}(Q)$ is finitely embedded and $L$ can be naturally embedded in $\bigoplus_{i=1}^{s} M / f_{i}^{-1}(Q)$, it turns out that $L$ is finitely embedded. Let $\pi: M \longrightarrow L$ denote the natural epimorphism. The exact sequence

$$
0 \longrightarrow K \stackrel{\mu}{\longrightarrow} F \stackrel{\rho}{\longrightarrow} N \longrightarrow 0
$$

yields the following commutative diagram:

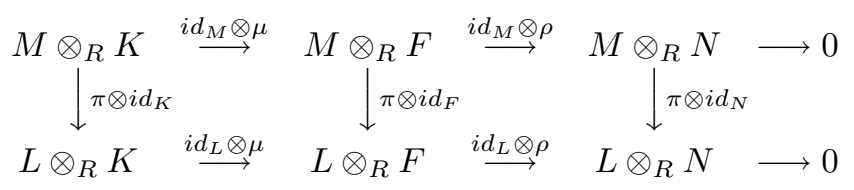

To show that $\left(\pi \otimes i d_{N}\right)(\alpha) \neq 0$, it is enough to see that $\left(\pi \otimes i d_{F}\right)(\beta) \notin i m\left(i d_{L} \otimes \mu\right)$. Suppose the contrary is true. Then there are $y_{1}, y_{2}, \ldots, y_{l} \in M$ and $z_{1}, z_{2}, \ldots, z_{l} \in$ $K$ such that

$$
\sum_{i=1}^{s}\left(\pi\left(m_{i}\right) \otimes x_{i}\right)=\left(\pi \otimes i d_{F}\right)(\beta)=\left(i d_{L} \otimes \mu\right)\left(\sum_{j=1}^{l}\left(\pi\left(y_{j}\right) \otimes z_{j}\right)\right) .
$$

For any given integer $1 \leq j \leq l$, let $r_{i j} \in R, i=1,2, \ldots, s$, be such that $z_{j}=$ $\sum_{i=1}^{s} r_{i j} x_{i}$. Thus

$$
\sum_{i=1}^{s}\left(\pi\left(m_{i}\right) \otimes x_{i}\right)=\sum_{i=1}^{s}\left(\left(\sum_{j=1}^{l} \pi\left(r_{i j} y_{j}\right)\right) \otimes x_{i}\right),
$$

as elements of $L \otimes_{R} F$. Therefore $m_{i}-\sum_{j=1}^{l} r_{i j} y_{j} \in \bigcap_{k=1}^{s} f_{k}^{-1}(Q)$ for all $i=1, \ldots, s$, and so

$$
\sum_{i=1}^{s}\left(m_{i} \otimes x_{i}\right)-\sum_{i=1}^{s}\left(\left(\sum_{j=1}^{l} r_{i j} y_{j}\right) \otimes x_{i}\right) \in Q
$$

Because $\sum_{j=1}^{l}\left(y_{j} \otimes z_{j}\right) \in i m\left(i d_{M} \otimes \mu\right) \subseteq Q$, it turns out that $\beta \in Q$. Therefore, we have achieved a contradiction.

Proposition 2.5. Let $M$ be an R-module and let $\left\{Q_{i}\right\}_{i \in I}$ denote the class of all finitely embedded quotient modules of $M$. Then there exists a pure homomorphism $M \longrightarrow \prod_{i \in I} Q_{i}$.

Proof. For each $i \in I$, let $\pi_{i}: M \longrightarrow Q_{i}$ denote the natural epimorphism. Define the homomorphism $\lambda: M \longrightarrow \prod_{i \in I} Q_{i}$ by $m \mapsto\left(\pi_{i}(m)\right)_{i \in I}$. To show that $\lambda$ is pure, it is enough to see that for any finitely presented $R$-module $N$, the $R$-homomorphism

$$
\lambda \otimes i d_{N}: M \otimes_{R} N \longrightarrow\left(\prod_{i \in I} Q_{i}\right) \otimes_{R} N
$$


is injective. Note that, by [3. Construction 18-2.6], any $R$-module is isomorphic to the direct limit of a family of finitely presented modules. It is a routine check to see that the map

$$
\Theta:\left(\prod_{i \in I} Q_{i}\right) \otimes_{R} N \longrightarrow \prod_{i \in I}\left(Q_{i} \otimes_{R} N\right),
$$

defined by $\left(y_{i}\right)_{i \in I} \otimes z \mapsto\left(y_{i} \otimes z\right)_{i \in I}$, is an isomorphism. Now, the conclusion follows, because by Lemma $2.4, \Theta\left(\lambda \otimes i d_{N}\right)$ is injective.

Let $D$ be a pure injective $R$-module and $f: D \longrightarrow L$ a pure homomorphism. Then, it follows that $D$ is isomorphic to a direct summand of $L$. This easy fact yields the following.

Corollary 2.6. Let $D$ be a pure injective $R$-module. Then $D$ is isomorphic to a direct summand of the direct product of a family of finitely embedded modules.

Example 2.7. By [5, Theorem 6], a Prüfer domain $R$ is locally almost maximal if and only if every finitely embedded $R$-module is pure injective. On other hand, by [2, Example 2.4] there exists a valuation domain $R$ such that $R$ is not almost maximal. Hence finitely embedded modules are not pure injective in general, and so the converse of Corollary 2.6 is not true.

Now, we are ready to establish our first characterization of pure injective modules.

Theorem 2.8. Let $R$ be a Noetherian ring and $D$ an $R$-module. Then $D$ is pure injective if and only if $D$ is isomorphic to a direct summand of the direct product of a family of Artinian modules.

Proof. First assume that $D$ is isomorphic to a direct summand of the direct product of a family of Artinian modules. By [6, Corollary 4.2], any Artinian module is pure injective. Thus $D$ is a pure injective module. Note that the direct product of a family $\left\{D_{i}\right\}_{i \in I}$ of $R$-modules is pure injective if and only if each $D_{i}$ is pure injective.

Now, assume that $D$ is pure injective. Then, it follows that $D$ is isomorphic to a direct summand of the direct product of a family of finitely embedded $R$-modules. But, over a Noetherian ring finitely embedded $R$-modules are Artinian.

Example 2.9. There is a pure injective module which is not the direct product of any family of Artinian modules. To this end, let $(R, \mathfrak{m})$ be a Noetherian complete local ring which is not Artinian. Since $R \cong \operatorname{Hom}_{R}(\mathrm{E}(R / \mathfrak{m}), \mathrm{E}(R / \mathfrak{m}))$, it follows by [4, Lemma 2.1] that $R$ is pure injective. Assume that there is a family $\left\{A_{i}\right\}_{i \in I}$ of Artinian modules such that $R=\prod_{i \in I} A_{i}$. Then only finitely many $A_{i}$ 's can be nonzero, because $R$ is Noetherian. Hence $R$ becomes an Artinian $R$-module, and so we achieved a contradiction.

Lemma 2.10. Let $\Omega$ denote the class of all R-algebras $T$ which are finitely presented as $R$-modules. Let $N$ be a submodule of an $R$-module $M$. Then the inclusion map $i: N \hookrightarrow M$ is pure if and only if $i \otimes i d_{T}$ is injective for all $T \in \Omega$.

Proof. It is enough to prove the "if" part. To this end, by [3, Construction 18-2.6], it will suffice to show that for any finitely presented $R$-module $L, i \otimes i d_{L}$ is injective. Let $L$ be a finitely presented $R$-module and $T_{L}=R \oplus L$ denote the trivial extension of $R$ by $L$. Recall that addition and multiplication in $T_{L}$ are defined, respectively, by

$$
(r, x)+\left(r^{\prime}, x^{\prime}\right)=\left(r+r^{\prime}, x+x^{\prime}\right)
$$


and

$$
(r, x)\left(r^{\prime}, x^{\prime}\right)=\left(r r^{\prime}, r x^{\prime}+r^{\prime} x\right) .
$$

Consider the ring homomorphism $\psi_{L}: R \longrightarrow T_{L}$ defined by $r \mapsto(r, 0)$. Clearly, the $R$-module structure on $T_{L}$ induced by $\psi_{L}$ coincides with the usual $R$-module structure of $T_{L}$ when $T_{L}$ is considered as the direct sum of two $R$-modules $R$ and $L$.

Let $\lambda: L \longrightarrow T_{L}$ be the natural embedding defined by $x \mapsto(0, x)$. Now, consider the following diagram:

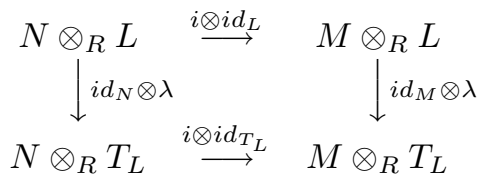

Because $\lambda$ is a pure $R$-homomorphism, we deduce that both maps $i d_{N} \otimes \lambda$ and $i d_{M} \otimes \lambda$ are injective. Thus we conclude that $i \otimes i d_{L}$ is injective, as required.

Proposition 2.11. Let $E$ be an injective cogenerator of $R$ and $D$ an $R$-module. Then $D$ is pure injective if and only if there is a family $\left\{T_{\lambda}\right\}_{\lambda \in \Lambda}$ of $R$-algebras which are finitely presented as $R$-modules, such that $D$ is isomorphic to a direct summand of $\prod_{\lambda \in \Lambda} \operatorname{Hom}_{R}\left(T_{\lambda}, E\right)$.

Proof. It is easy to see that for any $R$-module $M$ and any injective $R$-module $E^{\prime}$, the $R$-module $\operatorname{Hom}_{R}\left(M, E^{\prime}\right)$ is pure injective (see e.g. [4, Lemma 2.1]). Thus the "if" part follows clearly, because the direct product of a family $\left\{D_{i}\right\}_{i \in I}$ of $R$-modules is pure injective if and only if $D_{i}$ is pure injective for all $i \in I$.

Now, we prove the converse. Let $\Omega$ be as in Lemma 2.10. There is a subclass $\Omega^{*}$ of $\Omega$, which is a set, with the property that any $T \in \Omega$ is isomorphic (as an $R$-module) to an element of $\Omega^{*}$. Let $(.)^{\vee}$ denote the functor $\operatorname{Hom}_{R}(., E)$. Also, let $\Lambda$ be the set of all pairs $(T, f)$ with $T \in \Omega^{*}$ and $f \in \operatorname{Hom}_{R}\left(D, T^{\vee}\right)$, and for each $\lambda \in \Lambda$ denote the corresponding pair by $\left(T_{\lambda}, f_{\lambda}\right)$. Set $C=\prod_{\lambda \in \Lambda}\left(T_{\lambda}\right)^{\vee}$ and define the monomorphism $\psi: D \longrightarrow C$ by $\psi(x)=\left(f_{\lambda}(x)\right)_{\lambda \in \Lambda}$. By the paragraph preceding Corollary 2.6, the proof will be complete if we show that $\psi$ is pure. To this end, by Lemma 2.10, it is enough to show that for any $T \in \Omega^{*}$, the map $\psi \otimes i d_{T}$ is injective. Because the functor $(.)^{\vee}$ is faithfully exact, we equivalently prove that the natural $R$-homomorphism $\operatorname{Hom}_{R}\left(C, T^{\vee}\right) \longrightarrow \operatorname{Hom}_{R}\left(D, T^{\vee}\right)$ is surjective. Let $f \in \operatorname{Hom}_{R}\left(D, T^{\vee}\right)$. There is $\lambda_{0} \in \Lambda$ with $\left(T_{\lambda_{0}}, f_{\lambda_{0}}\right)=(T, f)$. Denote the projection $\operatorname{map} \prod_{\lambda \in \Lambda}\left(T_{\lambda}\right)^{\vee} \longrightarrow\left(T_{\lambda_{0}}\right)^{\vee}$, by $\rho_{\lambda_{0}}$. Then $\rho_{\lambda_{0}} \psi=f$.

Next, we present another characterization of pure injective modules.

Corollary 2.12. Let $D$ be an R-module. Then $D$ is pure injective if and only if $D$ is isomorphic to a direct summand of a module of the form $\operatorname{Hom}_{R}(L, E)$, where $E$ is an injective $R$-module and $L$ is the direct sum of a family of finitely presented $R$-modules.

Proof. The "if" part is clear, as we have seen in the proof of Proposition 2.11.

Now, let $D$ be a pure injective $R$-module and $E$ an injective cogenerator of $R$. Then, by Proposition 2.11, there is a family $\left\{L_{i}\right\}_{i \in I}$ of finitely presented $R$ modules such that $D$ is isomorphic to a direct summand of $\prod_{i \in I} \operatorname{Hom}_{R}\left(L_{i}, E\right)$. Set 
$L=\bigoplus_{i \in I} L_{i}$. Then

$$
\prod_{i \in I} \operatorname{Hom}_{R}\left(L_{i}, E\right) \cong \operatorname{Hom}_{R}(L, E),
$$

and so the conclusion follows.

Now, we are ready to present our second characterization of pure injective modules.

Theorem 2.13. An R-module $D$ is pure injective if and only if there is a family $\left\{T_{\lambda}\right\}_{\lambda \in \Lambda}$ of $R$-algebras which are finitely presented as $R$-modules, such that $D$ is isomorphic to a direct summand of a module of the form $\prod_{\lambda \in \Lambda} E_{\lambda}$, where for each $\lambda \in \Lambda, E_{\lambda}$ is an injective $T_{\lambda}$-module.

Proof. Again, the "if" part is clear, because as one can easily see, any pure injective module over an $R$-algebra $T$ is also pure injective as an $R$-module.

Now, we prove the converse. Let $E$ be an injective $R$-module and $T$ an $R$-algebra. Then, it is easy to see that $\operatorname{Hom}_{R}(T, E)$ is an injective $T$-module. Hence the claim follows, by Proposition 2.11 .

\section{REFERENCES}

[1] F.W. Anderson and K.R. Fuller, Rings and categories of modules, 2nd edition, SpringerVerlag, New York, 1992. MR1245487 (94i:16001)

[2] W. Brandal, Almost maximal integral domains and finitely generated modules, Trans. Amer. Math. Soc., 183 (1973), 203-222. MR0325609(48:3956)

[3] J. Dauns, Modules and rings, Cambridge University Press, Cambridge, 1994. MR.1301329 (95j:16001)

[4] E. Enochs, Flat covers and flat cotorsion modules, Proc. Amer. Math. Soc., 92(2) (1984), 179-184. MR0754698 (85j:13016)

[5] L. Fuchs and A. Meijer, Note on modules over Prüfer domains, Math. Pannon, 2(1) (1991), 3-11. MR:1119718 (92f:13021)

[6] L. Melkersson, Cohomological properties of modules with secondary representations, Math. Scand., 77(2) (1995), 197-208. MR1379266 (97d:13014)

[7] D.W. Sharpe and P. Vámos, Injective modules, Cambridge Tracts in Mathematics and Mathematical Physics, 62, Cambridge University Press, London-New York, 1972. MR0360706 (50:13153)

[8] R.B. Warfield, Purity and algebraic compactness for modules, Pacific J. Math., 28 (1969), 699-719. MR0242885 (39:4212)

[9] J. Xu, Flat covers of modules, Lecture Notes in Mathematics, 1634, Springer-Verlag, Berlin, 1996. MR1438789(98b:16003)

Department of Mathematics, Az-Zahra University, Vanak, Post Code 19834, Tehran, Iran - and - Institute for Studies in Theoretical Physics and Mathematics, P.O. Box 19395-5746, TeHran, Iran

E-mail address: kdivaani@ipm.ir

Department of Mathematics, Shahid Beheshti University, Tehran, Iran - and - Institute for Studies in Theoretical Physics and Mathematics, P.O. Box 19395-5746, Tehran, IRAN

Department of Mathematics, Shahid Beheshti University, Tehran, Iran - and - Institute for Studies in Theoretical Physics and Mathematics, P.O. Box 19395-5746, Tehran, IRAN 